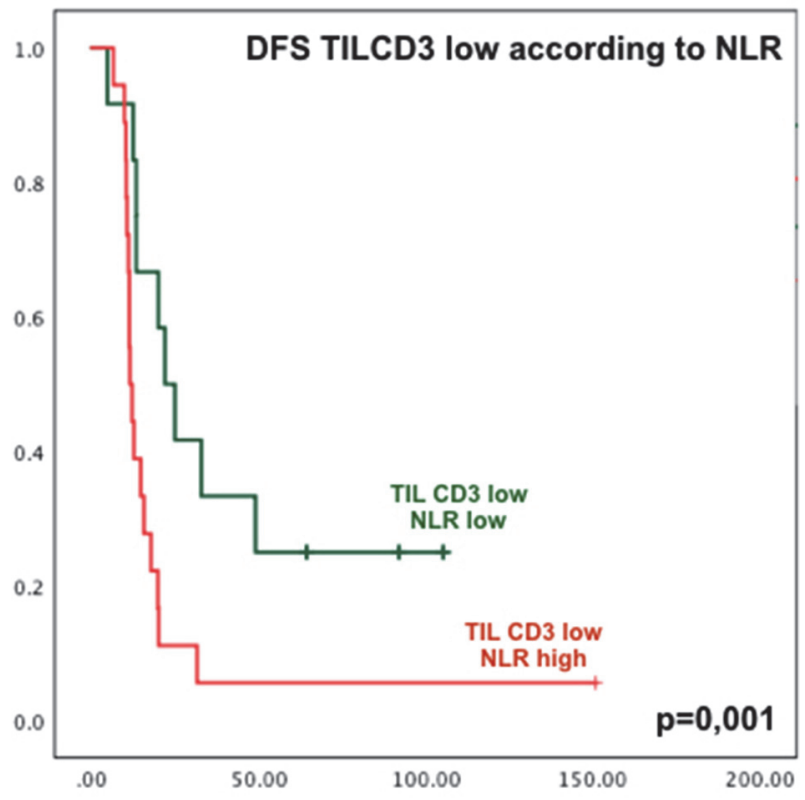

Abstract 413 Figure 2

$\mathrm{p}=0.057)$, and NLR $<3.72(\mathrm{p}=0.004)$ (figure 1$)$. The combined TIL- NLR analysis stratified patients with infiltration by TILCD3 $<25 \%$ into 2 subgroups with clearly different prognoses in terms of disease-free survival (DFS) (median DFS 11.49 vs 21.94 months HR 2.74 95\% CI 1.18 - 6.26; p =0.019) (figure 2). In addition, the combined analysis in the group of patients differentiated four different prognostic subgroups for OS ( $p=$ 0.05). The multivariate analysis including PS and histology showed an independent prognostic value in the variable TILNLR in OS $(p=0.009)$ and DFS ( $p=0.003)$.

Conclusion The combination of TILCD3 and NLR increases their prognostic value in OC. Combination prognostic factor could be very useful for improving immunotherapy strategies in advanced ovarian cancer.

Disclosures B. Álvarez-Abril: None. E. García: None. P. de la Morena: None. A. Ivars: None. M.Sánchez: None. A. Chaves: None. F. Pastor: None. G. Marín: None. F. Ayala de la Peña: None. E. García-Martínez: None.

\section{ELUCIDATING RESISTANCE MECHANISM TO PARP INHIBITORS FOR THE DEVELOPMENT OF NOVEL THERAPEUTIC APPROACHES IN HIGH-GRADE SEROUS OVARIAN CANCER}

\begin{abstract}
${ }^{1}$ Hagen Kulbe, ${ }^{2}$ Wanja Kassuhn, ${ }^{2}$ Frauke Ringel, ${ }^{2}$ Gabriele Welsch, ${ }^{2}$ Peggy Treffkorn, ${ }^{2}$ Eliane Taube, ${ }^{2}$ David Horst, ${ }^{2}$ Jalid Sehouli, ${ }^{2}$ Elena Braicu. ${ }^{1}$ Charité Universitätsmedizin Berlin; Department of Gynecology, Campus Virchow Clinic; ${ }^{2}$ Charité - Universitätsmedizin Berlin, Corporate Member of Freie Universität Berlin, Humboldt-Universität zu Berlin, and Berlin Institute of Health
\end{abstract}

\subsection{6/ijgc-2020-ESG0.133}

Introduction/Background PARP inhibitors (PARPi) have been established as a targeted therapeutic approach not only in patients with high-grade serous ovarian cancer (HGSOC) that have genetic loss of function of BRCA1/2-associated DNA repair. However, treatment efficacy varies and neither BRCA mutation, nor homolog recombination deficiency (HRD) status seem to be optimal predictors. Moreover, mechanisms of treatment resistance are poorly understood and novel approaches are urgently required.

Methodology Here we created gene expression data of HGSOC patients $(n=52)$ before PARPi treatment to elucidate key signaling pathways of resistance to increase their efficacy in combinatorial therapeutic strategies. We performed a comprehensive bioinformatics analysis of the differentially expressed genes between the $25 \%$ extreme responders $(n=26$; 13 each group), including gene set enrichment analysis (GSEA) and causal inference analysis with the CARNIVAL pipeline to elucidate the underlying molecular and regulatory mechanisms governing treatment efficacy and resistance.

Results In accordance with recent publications, we found higher levels of MYC activity in non-responders and deregulation of the $\mathrm{Wnt} / ß$-catenin signaling pathway resulting in PARPi treatment resistance. The pathway enrichment analysis also revealed specific pathways especially PDGFR, FGFR, PI3K/ mTOR and MAPK signaling pathway associated with resistant phenotype. Furthermore, we have identified key kinases, particularly JAK1/2 and SRC that might mediate resistance to PARP inhibition. In addition, differential gene expression analysis revealed folate receptor 1 (FOLR1) to be significantly higher expressed in non-responders $(\log \mathrm{FC}=2.66 ; \mathrm{p}<$ 0.0026) with the potential as a serum-based biomarker not only for ovarian cancer, as it correlates closely with CA125, but also PARPi treatment efficacy.

Conclusion In conclusion, these findings define a network of pathways, that are crucial to mediate mechanism of PARPi resistance and identified key signaling kinases as therapeutic targets in ovarian cancer.

Disclosures The authors declare no conflict of interest.

\section{FIRST REAL-WORLD HEMATOLOGIC ADVERSE EVENTS EXPERIENCE WITH NIRAPARIB IN ADVANCED OVARIAN CANCER}

Junjian Wang, Jianqing Zhu. Cancer Hospital of the University of Chinese Academy of Sciences (zhejiang Cancer Hospital)

\subsection{6/ijgc-2020-ESG0.134}

Introduction/Background Niraparib, a poly (ADP-ribose) polymerase (PARP) 1/2 inhibitor, has been approved by Food and Drug Administration (FDA) for $\geq 3$ line recurrence ovarian cancer (OC), the platinum-sensitive recurrence maintenance treatment and new diagnosed maintenance treatment using individual starting dose (ISD, $200 \mathrm{mg}$ daily for body weight $<77 \mathrm{~kg}$ or platelet count $<150,000 / \mu \mathrm{L}$, $300 \mathrm{mg}$ daily for body weight $\geq 77 \mathrm{~kg}$ and $\geq 150,000 / \mu \mathrm{L}$ ). This study aimed to retrospectively assess the incidence of hematologic adverse events (AEs) in real-world Chinese OC patients using ISD niraparib in Zhejiang Cancer Hospital.

Methodology All medical records of OC patients with ISD niraparib in the Zhejiang Cancer Hospital from February 2019 to January 2020 were reviewed. Treatment-emergent hematologic AEs including leukopenia, anemia and thrombocytopenia were collected and analyzed.

Results A total of 43 patients with OC were included in this study. The median body weight was $50.5(33,75) \mathrm{kg} .200$ mg QD was taken as ISD for all patients. Twenty seven $(62.8 \%)$ patients were of BRCA wild-type, 14 (32.6\%) were of BRCA mutants and $2(4.6 \%)$ were unknown. Niraparib 
was used as newly diagnosed maintenance treatment in 25 (58.1\%) patients, as treatment for $\geq 3$ line recurrence treatment in $14(32.6 \%)$ patients, and as platinum-sensitive recurrent maintenance treatment in $4(9.3 \%)$ patients. Overall, 28 (65.1\%) patients experienced $\geq 1$ grade hematologic AEs, which included leukopenia (37.2\%), anemia (34.9\%) and thrombocytopenia (39.5\%). Only $10 \quad(23.3 \%)$ patients had grade 3/4 AEs including leukopenia (9.3\%), anemia (7.0\%) and thrombocytopenia (11.6\%). Until last follow up, the median time for the occurrence of leukopenia, anemia and thrombocytopenia were 30 (range: 7, 162), 34 (range: 7, 108) and 20 (range: 13, 180) days, respectively. No deaths were reported. Of those patients who experienced AEs during treatment, the dose was reduced in $4(14.3 \%)$ patients, and treatment was interrupted in $9(32.1 \%)$ patients. Additional recombinant human granulocyte colony stimulating factor $(n=5,17.9 \%)$, erythrocyte $(n=2,7.1 \%)$ and recombinant human thrombopoietin $(n=5,17.9 \%)$ were provided for treating the AEs. After intervention, $8(18.6 \%)$ patients restart the treatment and only $1(2.3 \%)$ patient discontinued the treatment.

Conclusion The incidence of hematologic AEs in real-world experience was lower than reported by niraparib $300 \mathrm{mg} /$ day in ENGOT-OV16/NOVA trial. In addition to maintenance treatment in the first line, the patients in platinum-sensitive recurrence treatment and later line treatment might benefit from ISD niraparib.

Disclosures The authors declare that they have no competing interests.

\section{SIGNALING PATHWAYS RELATED WITH ITGBL1 IN OVARIAN CANCER CELLS}

${ }^{1}$ Alexander J Cortez, ${ }^{2}$ Katarzyna A Kujawa, ${ }^{3}$ Agata M Wilk, ${ }^{3}$ Marcela K Krzempek, ${ }^{2}$ Joanna P Syrkis, ${ }^{2}$ Magdalena Olbryt, ${ }^{2}$ Katarzyna M Lisowska. ${ }^{1}$ Maria Sklodowska-Curie National Research Institute of Oncology, Gliwice Branch; Department of Biostatistics and Bioinformatics, Center for Translational Research and Molecular Biology of Cancer; ${ }^{2}$ Maria Sklodowska-Curie National Research Institute of Oncology, Gliwice Branch; Center for Translational Research and Molecular Biology of Cancer; ${ }^{3}$ Maria Sklodowska-Curie National Research Institute of Oncology, Gliwice Branch; Department of Biostatistics and Bioinformatics

\subsection{6/ijgc-2020-ESG0.135}

Introduction/Background Integrin beta-like 1 (ITGBL1) is a poorly characterized protein comprised of ten EGF-like repeats. Our previous studies suggested that higher ITGBL1 mRNA expression level in the tumor is related with shorter survival of ovarian cancer patients. ${ }^{1}{ }^{2}$ Subsequent functional in vitro studies revealed that ITGBL1 overexpression in ovarian cancer cells resulted in the altered adhesion, migration and invasiveness, while it had no effect on proliferation rate and the cell cycle. ITGBL1-overexpressing cells were significantly more resistant to cisplatin and paclitaxel, ${ }^{3}$ major drugs used in OC treatment. ${ }^{4}$ In the current study we analyzed gene expression profiles of ITGBL1-overexpressing and control ovarian cancer cells and investigated ITGBL1 influence on ovarian cancer cell signaling pathways.

Methodology ITGBL1 coding sequence was PCR-amplified from cDNA and cloned into pLNCX2 vector. Retroviral system was used to obtain two ovarian cancer cell lines: OAW42/ITGBL1(+) and SKOV3/ITGBL1(+) with overexpression of ITGBL1. Control cell lines were obtained by transduction with an empty vector. RNA was isolated from wild type,
ITGBL1-overexpressing and control cells. DNA microarray experiment was performed using GeneChip ${ }^{\text {тм }}$ Human Transcriptome Array 2.0 (Affymetrix, Santa Clara, CA, USA) according to the manufacturer's instructions. Bioinformatical analysis was carried out in $\mathrm{R}$ environment (version 3.5.3) with Bioconductor packages.

Results Using Principal Component Analysis, an unsupervised method of data analysis, we selected gene sets related to major sources of variability in our dataset. Then, by performing Gene Set Enrichment Analysis we found 76 and 146 significantly affected cellular signaling pathways (in OAW42 and SKOV3 cell line, respectively). Majority of them (22 and 44, respectively) were related to extracellular matrix structure and function, integrin signaling, focal adhesion, cell junction, cellular motility, ERBB2 and ERBB4 signaling, etc.

Conclusion Global gene expression analysis revealed that signaling pathways affected by ITGBL1 overexpression were mostly those related to extracellular matrix organization and function, integrin signaling, focal adhesion, cellular communication and motility. These results are concordant with functional changes observed in ITGBL1-overexpressing cells, like altered adhesiveness, enhanced motility and invasiveness. Overall, our results indicate that higher expression of ITGBL1 in ovarian cancer cells is associated with features that may worsen clinical course of the disease.

A.J.Cortez was co-financed by the EU through the European Social Fund (grant-POWR.03.02.00-00-I029).

\section{REFERENCES}

1. Lisowska,et al.(2014),DOl:10.3389/fonc.2014.00006

2. Lisowska, et al.(2016),DOl:10.1007/s00432-016-2147-y

3. Cortez,et al.(2020),DOI: 10.3390/cancers12092676

4. Cortez,et al.(2020),DOI: 10.1007/s00280-017-3501-8

Disclosures Authors have nothing to disclose.

\section{FIBRONECTIN AND PERIOSTIN AS PROGNOSTIC MARKERS IN OVARIAN CANCER}

${ }^{1}$ Katarzyna A Kujawa, ${ }^{2}$ Ewa Zembala-Nożyńska, ${ }^{3}$ Alexander J Cortez, ${ }^{4}$ Jolanta Kupryjańczyk ${ }^{5}$ Katarzyna M Lisowska. 'Maria Sklodowska-Curie National Research Institute of Oncology, Gliwice Branch; Center for Translational Research and Molecular Biology of Cancer; ${ }^{2}$ Maria Sklodowska-Curie National Research Institute of Oncology, Gliwice Branch; Tumor Pathology Department; ${ }^{3}$ Maria Sklodowska-Curie National Research Institute of Oncology, Gliwice Branch; Department of Biostatistics and Bioinformatics, Center for Translational Research and Molecular Biology of Cancer; ${ }^{4}$ Maria Skłodowska-Curie Memorial Cancer Center and Institute of Oncology; Tumor Pathology Department; ${ }^{5}$ Maria Sklodowska-Curie National Research Institute of Oncology, Gliwice Branch; Center for Translational Research and Molecular Biology of Cancer

\subsection{6/ijgc-2020-ESG0.136}

Introduction/Background In our previous microarray study we identified a 96-gene prognostic signature associated with the shorter overall survival (OS) of ovarian cancer patients. ${ }^{1}$ Two genes from this signature, both coding for extracellular matrix proteins, were objects of the present study: FN1 and POSTN. We analyzed, by immunostaining, expression of encoded proteins in the independent set of ovarian cancer samples and evaluated its correlation with clinical, pathological, and molecular features.

Methodology Ovarian cancer samples from 108 patients were analyzed by immunochistochemistry using rabbit anti-human fibronectin polyclonal antibody (1:3000 dilution, A0245, Dako, Glostrup, USA) and rabbit anti-human periostin 